\title{
An asymptotically optimal kernel combined classifier
}

\author{
Majid Mojirsheibani ${ }^{1}$ and Jiajie $\mathrm{Kong}^{2}$ \\ Department of Mathematics, California State University Northridge, CA, 91330, USA
}

\begin{abstract}
A kernel ensemble classifier is developed for accurate classification based on several initial classifiers. A data-driven choice of the smoothing parameter of the kernel is considered and the resulting classifier is shown to be asymptotically optimal. Therefore, the proposed combined classifier asymptotically outperforms each individual classifier.
\end{abstract}

Keywords: Kernel, Hamming distance, smoothing parameter, combined classifier.

\section{Introduction}

This article focuses on methods and procedures that can be used to combined several individual classifiers in order to develop more effective classification rules. Different classifiers are usually constructed based on different theory or different estimation procedures and, depending on the type of distributional assumptions imposed on the data, some classifiers perform better in the sense of having lower misclassification error rates. However, given a number of individual classifiers, it is not always clear as to how to develop a systematic approach or a general framework for choosing the classifier with the smallest error rate; this is particularly true in nonparametric situations. Furthermore, since the exact distribution of the data is virtually always unknown, and since each classifier may have certain unique merits, it would be logical to somehow combine the individual classifiers in such a way that the resulting combined classifier would be superior. Combined or ensemble classification results in the literature may be divided into two main types: (i) those that start with a large number of base classifiers, which are usually homogeneous in nature (such as a large number of tree classifiers), which will then be combined into a final classification rule. (ii) Those that combine a number of classifiers that have been constructed using different theory or estimation procedures, which is the main focus of this paper. Breiman's $(1996,2001)$ popular Random Forests classifier falls under (i). Other results that fall under (i) include Biau et al.

\footnotetext{
${ }^{1}$ Corresponding author. Email: majid.mojirsheibani@csun.edu

This work is supported by the NSF Grant DMS-1407400 of Majid Mojirsheibani.

${ }^{2}$ Email: jiajie.kong.595@my.csun.edu
} 
(2008) and Lin and Jeon (2006). On the other hand, the work of LeBlanc and Tibshirani (1996), Mojirsheibani (1999), and Mojirsheibani and Montazeri (2015) fall primarily under (ii). For more on the taxonomy of ensemble classifiers one may refer to, for example, Zhang and Duin (2011).

There are many intuitively appealing methods for combining classifiers in the literature; these methods may be put into two categories themselves: weighting methods and metalearning. Weighting methods include the majority voting approach used, for example, by Breiman (1996, 2001) for tree classification, and by Xu et al. (1992) in handwriting recognition. Averaging and weighted-averaging of estimated class conditional probabilities (that are produced by each individual classifier) have also been considered by some authors; see, for example, Xu et al. (1992), Breiman (1995), and LeBlanc and Tibshirani (1996). The work of Adler et al. (2011) on combined classification for paired data, and De Bock et al. (2010) on a generalized additive model based approach to ensembles provide additional new directions. There are also other weighting methods that can be found in Rokach (2009, 2010). Meta-learning methods usually work by using the predicted values of the individual classifiers on the data. Relevant results along these lines include the stacked generaliztion of Wolpert (1992), Breiman's (1995) stacked method, and Mojirsheibani's (1999) nonlinear combined classifiers. For a detailed account of meta-learning methods one may refer to Rokach (2009). Combined or ensemble estimation has also been employed in regression and model selection problems in the literature. See, for example, the work of Yang $(2000,2004)$ and van der Laan et al. (2007). In a more recent article, Biau et al. (2015) proposed a combined regression estimator which can, asymptotically, outperform each of the individual regression estimators in the $L_{2}$ sense.

The rest of the paper is organized as follows. In Section 2 we discuss and study kernel combined classifiers; our key contributions appear in Theorem 2. In Section 2.2 we carry out some numerical studies that confirm our theoretical findings. All proofs are deferred to an Appendix in Section 3. 


\section{The proposed kernel combined classifier}

\subsection{Methodology}

In this article we consider the following standard two-group classification problem. Let $(\mathbf{X}, Y)$ be a random pair, where $\mathbf{X} \in \mathbb{R}^{d}$ is called the covariate or feature vector and $Y \in$ $\{0,1\}$, called the class variable, has to be predicted based on $\mathbf{X}$. The aim of classification is to find a function (a classifier) $\psi: \mathbb{R}^{d} \rightarrow\{0,1\}$ whose misclassification error rate (i.e., the probability of incorrect prediction), $P\{\psi(\mathbf{X}) \neq Y\}$, is as small as possible. The best classifier, also called the Bayes classifier, is given by

$$
\psi_{B}(\mathbf{X})= \begin{cases}1 & \text { if } P\{Y=1 \mid \mathbf{X}\}>\frac{1}{2} \\ 0 & \text { otherwise }\end{cases}
$$

where $P\{Y=1 \mid \mathbf{X}\}=E[I\{Y=1\} \mid \mathbf{X}]$ and where $I\{C\}$ stands for the indicator function of the set $C$; see, for example, Devroye et al. (1996, Ch. 2). In practice the distribution of $(\mathbf{X}, Y)$ is always fully or partially unknown and therefore finding the function $\psi_{B}$ is impossible. Now suppose that we have available $n$ independently and identically distributed (iid) observations, i.e., the data, $\mathbb{D}_{n}:=\left\{\left(\mathbf{X}_{1}, Y_{1}\right), \cdots,\left(\mathbf{X}_{n}, Y_{n}\right)\right\}$, where $\left(\mathbf{X}_{i}, Y_{i}\right) \stackrel{\text { iid }}{=}(\mathbf{X}, Y), i=1, \cdots, n$, and let $\widehat{\psi}_{n, 1}, \ldots, \widehat{\psi}_{n, M}$ be $M$ different individual classifiers based on the data $\mathbb{D}_{n}$. For example, $\widehat{\psi}_{n, 1}$ may be a kernel classifier, $\widehat{\psi}_{n, 2}$ a nearest neighbor (NN) classifier, $\widehat{\psi}_{n, 3}$ might be something more complicated such as Breiman's (2001) random forests classifier or the support vector machines (SVM) of Boser et al. (1992), etc. Next let

$$
\widehat{\boldsymbol{\psi}}_{n}(\mathbf{X})=\left(\widehat{\psi}_{n, 1}(\mathbf{X}), \ldots, \widehat{\psi}_{n, M}(\mathbf{X})\right)^{\prime}
$$

be the vector of $M$ individual data-based classifiers and define the combined classifier $\phi_{n}^{*}$ by

$$
\phi_{n}^{*}\left(\widehat{\boldsymbol{\psi}}_{n}(\mathbf{X})\right)=\left\{\begin{array}{ll}
1 & \text { if } P\left\{Y=1 \mid \widehat{\boldsymbol{\psi}}_{n}(\mathbf{X})\right\}>\frac{1}{2} \\
0 & \text { otherwise. }
\end{array}= \begin{cases}1 & \text { if } E\left[(2 Y-1) \mid \widehat{\boldsymbol{\psi}}_{n}(\mathbf{X})\right]>0 \\
0 & \text { otherwise }\end{cases}\right.
$$

It is straightforward to show that $(2)$ is theoretically optimal in the sense that its overall misclassification error probability, $P\left\{\phi_{n}^{*}\left(\widehat{\boldsymbol{\psi}}_{n}(\mathbf{X})\right) \neq Y\right\}$, is less than or equal to that of any other combined classifier. More formally, we have the following elementary result

Theorem 1 The combined classifier $\phi_{n}^{*}$ in (2) is optimal, i.e.,

$$
P\left\{\phi_{n}^{*}\left(\widehat{\boldsymbol{\psi}}_{n}(\mathbf{X})\right) \neq Y\right\}=\inf _{\phi:\{0,1\}^{M} \rightarrow\{0,1\}} P\left\{\phi\left(\widehat{\boldsymbol{\psi}}_{n}(\mathbf{X})\right) \neq Y\right\}
$$


See the Appendix for a proof.

Of course, the above result immediately implies that for each individual classifier $\widehat{\psi}_{n, j}, j=$ $1, \ldots, M$, we have $P\left\{\phi_{n}^{*}\left(\widehat{\boldsymbol{\psi}}_{n}(\mathbf{X})\right) \neq Y\right\} \leq P\left\{\widehat{\psi}_{n, j}(\mathbf{X}) \neq Y\right\}$. Unfortunately the combined classifier $\phi_{n}^{*}$ in (2) is not available in practice because it depends on unknown conditional probabilities. In what follows we propose a kernel estimator of $\phi_{n}^{*}$, where the smoothing parameter of the kernel is estimated by a data-driven choice that minimizes the so-called resubstitution estimate of the probability of misclassification. We recall that for any classifier $\psi_{n}$, constructed based on the data $\left(\mathbf{X}_{i}, Y_{i}\right), i=1, \ldots, n$, the resubstitution estimator of the misclassification probability of $\psi_{n}$ is given by $n^{-1} \sum_{i=1}^{n} I\left\{\psi_{n}\left(\mathbf{X}_{i}\right) \neq Y_{i}\right\}$. Our proposed approach uses a data-splitting method that works as follows. Start by randomly splitting the data $\mathbb{D}_{n}:=\left\{\left(\mathbf{X}_{1}, Y_{1}\right), \ldots,\left(\mathbf{X}_{n}, Y_{n}\right)\right\}$ into two parts, $\mathbb{D}_{m}$ of size $m$ and $\mathbb{D}_{\ell}$ of size $\ell$, where $\ell+m=n$ and $\mathbb{D}_{m} \cup \mathbb{D}_{\ell}=\mathbb{D}_{n}$. Also, let $\widehat{\psi}_{m, 1}, \cdots, \widehat{\psi}_{m, M}$ be the individual classifiers constructed based on the subsample $\mathbb{D}_{m}$ only (instead of $\mathbb{D}_{n}$ ). Now, consider the class $\Phi_{n}$ of kernel combined classifiers of the form

$$
\phi_{n, h}\left(\widehat{\boldsymbol{\psi}}_{m}(\mathbf{X})\right)= \begin{cases}1 & \text { if } \sum_{i:\left(\mathbf{X}_{i}, Y_{i}\right) \in \mathbb{D}_{\ell}}\left(2 Y_{i}-1\right) \cdot \mathcal{K}\left(\frac{1}{h} d_{\mathrm{H}}\left(\widehat{\boldsymbol{\psi}}_{m}(\mathbf{X}), \widehat{\boldsymbol{\psi}}_{m}\left(\mathbf{X}_{i}\right)\right)\right)>0 \\ 0 & \text { otherwise }\end{cases}
$$

with the convention that $0 / 0:=0$ (thus $\mathcal{K}\left(\frac{0}{0}\right):=\mathcal{K}(0)$ ), where $d_{\mathrm{H}}$ is the Hamming distance (i.e., the number of disagreements between the components of the vectors $\widehat{\boldsymbol{\psi}}_{m}(\mathbf{X})$ and $\left.\widehat{\boldsymbol{\psi}}_{m}\left(\mathbf{X}_{i}\right)\right)$. Here $\widehat{\boldsymbol{\psi}}_{m}(\mathbf{X})=\left(\widehat{\psi}_{m, 1}(\mathbf{X}), \ldots, \widehat{\psi}_{m, M}(\mathbf{X})\right)^{\prime}$, and $\mathcal{K}:[0, \infty) \rightarrow[0, \infty)$ is the kernel used with the smoothing parameter $h \geq 0$. We observe that if we put $r_{\ell}\left(\widehat{\boldsymbol{\psi}}_{m}(\mathbf{X})\right)=$ $\sum_{i:\left(\mathbf{X}_{i}, Y_{i}\right) \in \mathbb{D}_{\ell}}\left(2 Y_{i}-1\right) \cdot \mathcal{K}\left(\frac{1}{h} d_{\mathrm{H}}\left(\widehat{\boldsymbol{\psi}}_{m}(\mathbf{X}), \widehat{\boldsymbol{\psi}}_{m}\left(\mathbf{X}_{i}\right)\right)\right) / \sum_{i:\left(\mathbf{X}_{i}, Y_{i}\right) \in \mathbb{D}_{\ell}} \mathcal{K}\left(\frac{1}{h} d_{\mathrm{H}}\left(\widehat{\boldsymbol{\psi}}_{m}(\mathbf{X}), \widehat{\boldsymbol{\psi}}_{m}\left(\mathbf{X}_{i}\right)\right)\right)$, then for each $h$, the quantity $r_{\ell}\left(\widehat{\boldsymbol{\psi}}_{m}(\mathbf{X})\right)$ is our kernel regression estimator of $E[(2 Y-$ 1) $\left.\mid \widehat{\psi}_{n}(\mathbf{X})\right]$ based on one sample split. Thus, (3) is equivalent to a classifier that assigns $\mathbf{X}$ to class 1 if $r_{\ell}\left(\widehat{\boldsymbol{\psi}}_{m}(\mathbf{X})\right)>0$. Since this estimator is based on one sample split only, the behavior of the classifier (3) can depend on the specific split used and, unless $n$ is very large, a "bad" split can result in the poor performance of (3). To overcome this in numerical applications, we recommend using several splits to replace $r_{\ell}\left(\widehat{\boldsymbol{\psi}}_{m}(\mathbf{X})\right)$ by the average of several of its values (each coming from a different split). In passing, we also note that when $h=0, \mathcal{K}(0)>0$, and $\lim _{t \rightarrow \infty} \mathcal{K}(t)=0$, the above rule reduces to the combined classifier of Balakrishnan and Mojirsheibani (2015), i.e., the combined classifier

$$
\phi_{n, 0}\left(\widehat{\boldsymbol{\psi}}_{m}(\mathbf{X})\right)= \begin{cases}1 & \text { if } \sum_{i:\left(\mathbf{X}_{i}, Y_{i}\right) \in \mathbb{D}_{\ell}}\left(2 Y_{i}-1\right) I\left\{\widehat{\boldsymbol{\psi}}_{m}(\mathbf{X})=\widehat{\boldsymbol{\psi}}_{m}\left(\mathbf{X}_{i}\right)\right\} \mathcal{K}(0)>0 \\ 0 & \text { otherwise }\end{cases}
$$




$$
= \begin{cases}1 & \text { if } \frac{\sum_{i:\left(\mathbf{x}_{i}, Y_{i}\right) \in \mathbb{B}_{\ell}} I\left\{Y_{i}=1, \widehat{\boldsymbol{\psi}}_{m}\left(\mathbf{X}_{i}\right)=\widehat{\boldsymbol{\psi}}_{m}(\mathbf{X})\right\}}{N_{m, \ell}(\mathbf{X})}>\frac{1}{2} \\ 0 & \text { otherwise, }\end{cases}
$$

where

$$
N_{m, \ell}(\mathbf{X})=\sum_{i: \mathbf{X}_{i} \in \mathbb{D}_{\ell}} I\left\{\widehat{\boldsymbol{\psi}}_{m}\left(\mathbf{X}_{i}\right)=\widehat{\boldsymbol{\psi}}_{m}(\mathbf{X})\right\} .
$$

The combined classifier of Balakrishnan and Mojirsheibani (2015) in (4) lacks smoothness in the sense that the individual classifiers must all agree at both $\mathbf{X}_{i}$ and the new $\mathbf{X}$ in order to have a "vote" of 1 from the indicator function $I\left\{\widehat{\boldsymbol{\psi}}_{m}\left(\mathbf{X}_{i}\right)=\widehat{\boldsymbol{\psi}}_{m}(\mathbf{X})\right\}$. Unfortunately, if there are some weak or poor classifiers among $\widehat{\psi}_{m, 1}, \cdots, \widehat{\psi}_{m, M}$, then $I\left\{\widehat{\boldsymbol{\psi}}_{m}\left(\mathbf{X}_{i}\right)=\widehat{\boldsymbol{\psi}}_{m}(\mathbf{X})\right\}$ can become 0 for a large number of $\mathbf{X}_{i} \in \mathbb{D}_{\ell}, i=1, \ldots, \ell$ (which can happen even if $\mathbf{X}$ and $\mathbf{X}_{i}$ both belong to the same class), and this can reduce the predictive performance of (4). In fact, a close examination of some of our numerical results revealed that for many new observations, $\mathbf{X}$, the sum $\sum_{i:\left(\mathbf{X}_{i}, Y_{i}\right) \in \mathbb{D}_{\ell}} I\left\{Y_{i}=1, \widehat{\boldsymbol{\psi}}_{m}\left(\mathbf{X}_{i}\right)=\widehat{\boldsymbol{\psi}}_{m}(\mathbf{X})\right\}$ is 0 only because of one or two poor classifiers (the LDA and the 5 -NN rules). As a result, for all such covariates $\mathbf{X}$, the combined classifier (4) assigns $\mathbf{X}$ to class 0 , which is worse than random guessing. On the other hand, since the events $\left\{I\left\{\widehat{\boldsymbol{\psi}}_{m}\left(\mathbf{X}_{i}\right)=\widehat{\boldsymbol{\psi}}_{m}(\mathbf{X})\right\}=0\right\}$ and $\left\{d_{\mathrm{H}}\left(\widehat{\boldsymbol{\psi}}_{m}(\mathbf{X}), \widehat{\boldsymbol{\psi}}_{m}\left(\mathbf{X}_{i}\right)\right) \geq 1\right\}$ are equivalent, the presence of the kernel $\mathcal{K}$ in (3) introduces smoothness by assigning a weight, other than 0 or 1 , to each observation, where the weight is inversely proportional to the magnitude of the Hamming distance between the vectors $\widehat{\boldsymbol{\psi}}_{m}(\mathbf{X})$ and $\widehat{\boldsymbol{\psi}}_{m}\left(\mathbf{X}_{i}\right)$ whenever $\mathcal{K}$ is a decreasing function. Thus, unlike the combined classifier (4) of Balakrishnan and Mojirsheibani (2015), the new combined classifier in (3) is not heavily affected by the influence of a few weak classifiers.

Next, let $\widehat{\phi}_{n}$ be the classifier chosen from the class $\Phi_{n}$ (of classifiers of the form (3)) that minimizes the resubstitution error

$$
\widehat{\mathcal{R}}_{\ell}\left(\phi_{n, h}\right)=\frac{1}{\ell} \sum_{i:\left(\mathbf{X}_{i}, Y_{i}\right) \in \mathbb{D}_{\ell}} I\left\{\phi_{n, h}\left(\widehat{\boldsymbol{\psi}}_{m}\left(\mathbf{X}_{i}\right)\right) \neq Y_{i}\right\}, \phi_{n, h} \in \Phi_{n}
$$

in other words,

$$
\widehat{\phi}_{n}=\underset{\phi_{n, h} \in \Phi_{n}}{\operatorname{argmin}} \widehat{\mathcal{R}}_{\ell}\left(\phi_{n, h}\right) .
$$

How good is the combined classifier $\widehat{\phi}_{n}$ as compared to the original individual classifiers $\widehat{\psi}_{n, 1}, \ldots, \widehat{\psi}_{n, M}$, (each of which is constructed based on the entire data of size $\left.n\right)$ ? We will 
show that, under rather minimal conditions, $\widehat{\phi}_{n}$ is asymptotically at least as good as the best individual classifier. To state our main results we also need the following assumptions:

(A1) The kernel $\mathcal{K}:[0, \infty) \rightarrow[0, \infty)$ satisfies: $K(0)>0$ and $\lim _{t \rightarrow \infty} K(t)=0$.

(A2) $N_{m, \ell}(\mathbf{X}) \rightarrow \infty$, in probability, as $n \rightarrow \infty$, where $N_{m, \ell}(\cdot)$ is given by (5).

(A3) $P\left\{\phi_{n}^{*}\left(\widehat{\boldsymbol{\psi}}_{n}(\mathbf{X})\right) \neq Y\right\} \rightarrow c$, as $n \rightarrow \infty$, for some $c \in[0,1]$, where $\phi_{n}^{*}$ is as in (2).

Condition (A2) is not as restrictive as it might appear. In fact, we observe that conditional on $\mathbb{D}_{m}$, the quantity $N_{m, \ell}(\mathbf{x})$ is simply the number of "successes" in $\ell$ independent Bernoulli trials which diverges for each $\mathbf{x}$, as $\ell$ goes to infinity. Versions of assumption (A2) have already been employed in the literature; see, for example, Devroye et al. (1996, Sec. 6.3). Our main results may be summarized as follows. Let $\widehat{\phi}_{n}$ and $\phi_{n}^{*}$ be as in (7) and (2), respectively, and define the misclassification error probabilities

$$
\mathcal{R}\left(\widehat{\phi}_{n}\right)=P\left\{\widehat{\phi}_{n}\left(\widehat{\boldsymbol{\psi}}_{m}(\mathbf{X})\right) \neq Y\right\} \text { and } \mathcal{R}\left(\phi_{n}^{*}\right)=P\left\{\phi_{n}^{*}\left(\widehat{\boldsymbol{\psi}}_{n}(\mathbf{X})\right) \neq Y\right\} \text {. }
$$

Theorem 2 Let $\widehat{\phi}_{n}$ be the resubstitution based kernel combined classifier defined via (7). Then, under assumptions (A1), (A2), and (A3), we have

$$
\mathcal{R}\left(\widehat{\phi}_{n}\right)-\mathcal{R}\left(\phi_{n}^{*}\right) \longrightarrow 0 \text {, as } n \rightarrow \infty
$$

where $\phi_{n}^{*}$ is the theoretically optimal combined classifier defined in (2).

(ii) $\widehat{\phi}_{n}$ is asymptotically at least as good as the best individual classifier, i.e.,

$$
\limsup _{n \rightarrow \infty} \max _{1 \leq j \leq M}\left\{\mathcal{R}\left(\widehat{\phi}_{n}\right)-\mathcal{R}\left(\widehat{\psi}_{n, j}\right)\right\} \leq 0,
$$

where $\widehat{\psi}_{n, j}$ is the $j$-th individual classifier constructed based on all of the data $\mathbb{D}_{n}$ and $\left.\mathcal{R}\left(\widehat{\psi}_{n, j}\right)=P\left\{\widehat{\psi}_{n, j}(\mathbf{X})\right) \neq Y\right\}$ is the misclassification error of $\widehat{\psi}_{n, j}$.

See the Appendix for a proof.

According to Theorem 2(ii), the error of the proposed combined classifier $\widehat{\phi}_{n}$ is, asymptotically, at least as low as that of the best initial classifier in the pool. Furthermore, Theorem 2(ii) implies that if any one of the constituent classifiers is already consistent (i.e., its error rate converges to that of the Bayes classifier), then so is the combined classifier. This, of course, does not mean that for each $n$ the error of $\widehat{\phi}_{n}$ is always significantly smaller than that of each individual classifier. In particular, if one (or more) of the classifiers is a strong performer, and possibly consistent, then the gain in error reduction may be rather small. On the other hand, when the initial classifiers are weak/poor, then the benefits gained from combined classification can be substantial. The following numerical examples shed more light on the performance of the proposed combined classifier under different situations. 


\section{$2.2 \quad$ Numerical examples}

In the following examples, we consider the prediction of the class variable, $Y=0$ or 1 , of the vector of covariates $\mathbf{X} \in \mathbb{R}^{5}$, under 3 different setups, where each setup signifies certain facts about the proposed combined classifier.

Example $A$.

Here $P\{Y=1\}=P\{Y=0\}=0.5$, where if $Y=1$ (i.e., class 1 ) then $\mathbf{X} \sim N_{5}(\mathbf{0}, \boldsymbol{\Sigma})$, with $\boldsymbol{\Sigma}=\left(\sigma_{i j}\right)_{i, j=1, \cdots, 5}$, and $\sigma_{i j}=2^{3-|i-j|}$. If $Y=0$ (i.e., class 0 ) then $\mathbf{X}$ has a 5 -dim standard Cauchy distribution with independent components, i.e., the components of $\mathbf{X}$ are independent standard Cauchy random variables. As for the choice of the individual classifiers, we have considered the following 6 classifiers: (i) The Support Vector Machine (SVM) of Boser et al. (1992), (ii) Breiman's (2001) Random Forests, (iii) a kernel classifier with the Gaussian kernel and a bandwidth of $n^{-0.1}$, (iv) a 5 Nearest Neighbor (5-NN) classifier, (v) a 1-NN classifier, and (vi) the Linear Discriminant Analysis (LDA). Next we considered five combined classifiers: $\widehat{\phi}_{n}$ defined via (7) and (6) where the kernel used in (3) is standard Gaussian where the search for the smoothing parameter is over the grid of equally spaced values $\{0,0.05,0.10,0.15, \ldots, 3.00\}$, the method of Balakrishnan and Mojirsheibani (2015) as given by (4), the majority-vote combined classifier (which is used in Breiman's (2001) Random Forests classifier), the combined classifier of Leblanc and Tibshirani (1996), and the combined classifier in Mojirsheibani (1999). The sample sizes used were $n=50,150,250,350$, 400, with $n / 2$ observations from each of the two distributions. For each value of $n$, we constructed the six individual classifiers which were then used to construct the five combined classifiers described above. To construct $\widehat{\phi}_{n}$ we took $m=\frac{2}{3} n$ and $\ell=\frac{1}{3} n$ for our sample splits. The fraction $\frac{2}{3}$ used here is not the only choice; in fact, any fraction in the interval $(0.5,0.8)$ produces nearly the same numerical results. Here we have used 20 sample splits in (3). Finally, to estimate the misclassification error probability of each classifier, we generated an additional sample of 1000 observations with 500 from each of the two distributions, to be used as a test sample by each classifier. The entire above process was repeated a total of 300 times resulting in 300 estimates of the misclassification error probabilities of each classifier. Table 1 summarizes the average error rates (over 300 Monte Carlo runs) for each classifier and several values of $n$. The numbers appearing in brackets are the standard errors over 300 Monte Carlo runs.

Table 1 shows that the three individual classifiers 1-NN, 5-NN, and LDA do not perform very well (the LDA is not even appropriate in this example). The SVM and Random Forests 
Table 1: Errors of the 5 combined classifiers and the 6 individual classifiers for Example A, where the first class is $N_{5}(\mathbf{0}, \boldsymbol{\Sigma}), \boldsymbol{\Sigma}=\left(\sigma_{i j}\right)_{i, j=1, \cdots, 5}$, where $\sigma_{i j}=2^{3-|i-j|}$, and the second class is a 5-dimensional standard Cauchy.

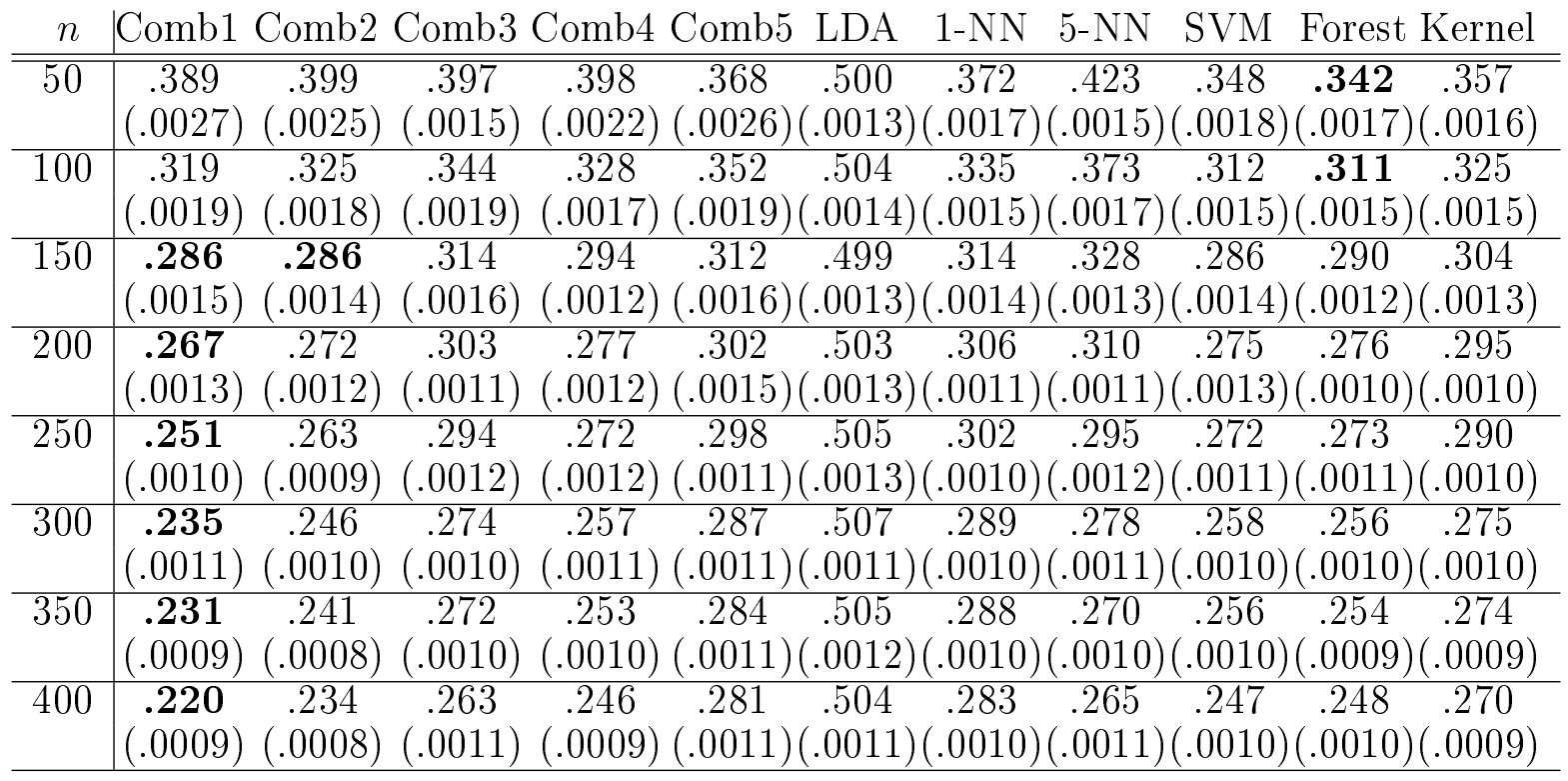

classifiers are the best individual classifiers (and have virtually the same performance). Table 1 also summarizes the performance of the 5 combined classifiers, which are denoted by Comb1 for the proposed combined classifier $\widehat{\phi}_{n}$ of this paper, Comb2 for the classifier of Balakrishnan and Mojirsheibani (2015), Comb3 for the majority-voting combined classifier, Comb4 for the method of Mojirsheibani (1999), and Comb5 for the method in Leblanc and Tibshirani (1996). The boldfaced numbers are the smallest error rates for each sample size. Interestingly, both SVM and Random Forests classifiers tend to perform very well for smaller sample sizes, but as $n$ increases to 150 , the proposed combined classifier Comb1, as well as Comb2, start outperforming all other classifiers.

Example B.

This is exactly the same as Example $A$ except that we are combining $M=3$ of the 6 classifiers; these are LDA, SVM, and Random Forests. The results appear in Table 2. Once again the proposed combined classifier outperforms the individual ones; however, unlike the results in Table 1, here the combined classifier Comb1 (and Comb2) is superior even when $n$ is as small as 50. In other words, the more individual classifiers are included in the ensemble (as in Example A), the larger $n$ should be to warrant good performance. For more on this, care for 
the remarks that appear at the end of Section 2. We also not that as in Example A, the error of the proposed combined classifier is only slightly better than that of SVM and Random Forests. This is because these two individual classifiers are excellent performers with error rates that may already be close to that of the Bayes classifier.

Table 2: Errors of the 5 combined classifiers and the 3 individual classifiers in Example $B$ where the first class is $N_{5}(\mathbf{0}, \boldsymbol{\Sigma}), \boldsymbol{\Sigma}=\left(\sigma_{i j}\right)_{i, j=1, \cdots, 5}$, with $\sigma_{i j}=2^{3-|i-j|}$, and the second class is a 5-dimensional standard Cauchy.

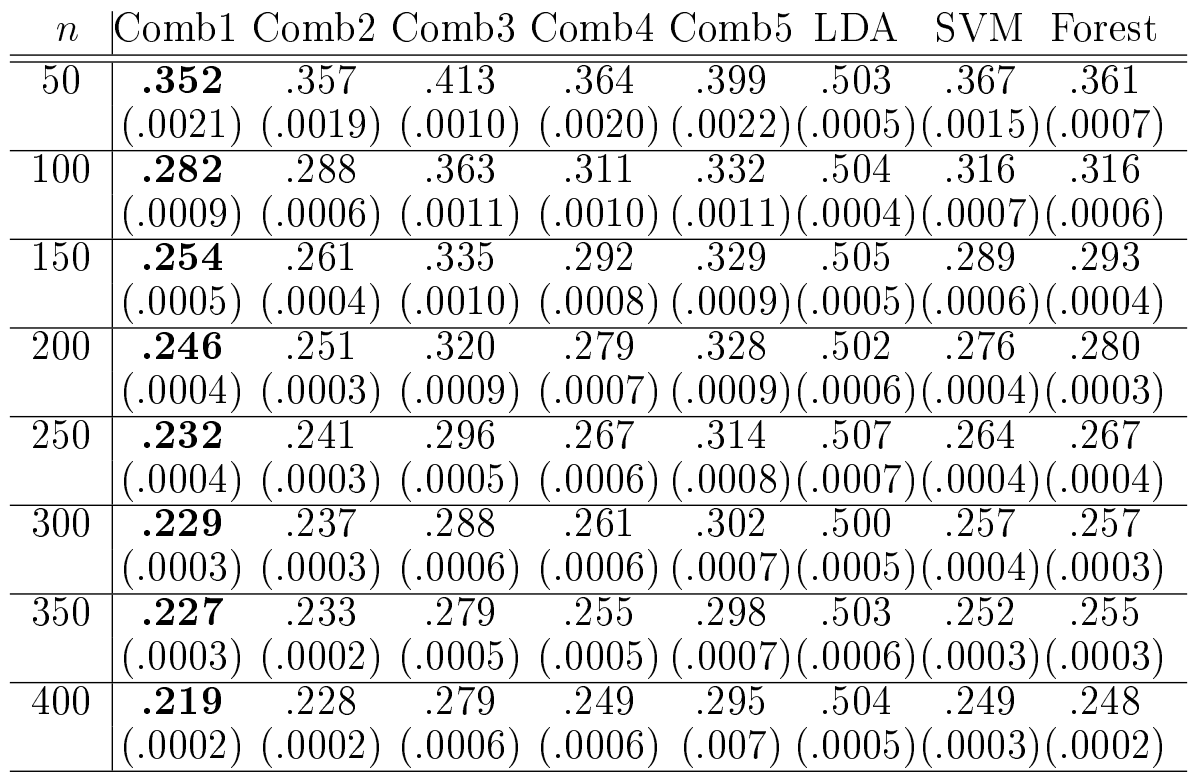

\section{Example C. [Combining several weak/poor classifiers.]}

Here the two class distributions are as in Example A except that the normal distribution (class 1) has covariance matrix $\boldsymbol{\Sigma}=\left(\sigma_{i j}\right)_{i, j=1, \cdots, 5}$, and $\sigma_{i j}=2^{-|i-j|}$. The five combined classifiers and the choice of the kernel $\mathcal{K}$ for the proposed classifier (Comb1) are as in Example $A$. As for the individual classifiers, the following five weak/poor classifiers are considered: the LDA, a 17-NN classifier, a 23-NN classifier, a kernel rule with $h=n^{-1.7}$, and a classifier, referred to as $C_{0}$, that assigns a new observation $\mathbf{X}_{\text {new }}$ to class 1 (Normal) if $\left\|\overline{\mathbf{X}}-\mathbf{X}_{\text {new }}\right\|>5$, (otherwise $\mathbf{X}_{\text {new }}$ is assigned to class 0 (Cauchy)), where $\overline{\mathbf{X}}$ is the sample mean of the data from class 1 (Normal). We note that $C_{0}$ is a poor classifier because, quite often, it confuses the two classes; to appreciate this, observe that if $\left\|\overline{\mathbf{X}}-\mathbf{X}_{\text {new }}\right\|>5$ then it makes more sense to assign $\mathbf{X}_{\text {new }}$ to class 0 (Cauchy) instead. We also note that the values $k=17$ and 23 are too large to make our NN rules useful. Finally, the LDA is inappropriate here whereas our 
kernel rule's bandwidth of $h=n^{-1.7}$ can be far too small to make it effective. As Table 3 shows, all five individual classifiers perform poorly.

Table 3: Errors of the 5 combined classifiers and the 5 weak/poor individual classifiers of Example $C$, where the first class is $N_{5}(\mathbf{0}, \boldsymbol{\Sigma}), \boldsymbol{\Sigma}=\left(\sigma_{i j}\right)_{i, j=1, \cdots, 5}$, where $\sigma_{i j}=2^{-|i-j|}$, and the second class is a 5-dimensional standard Cauchy.

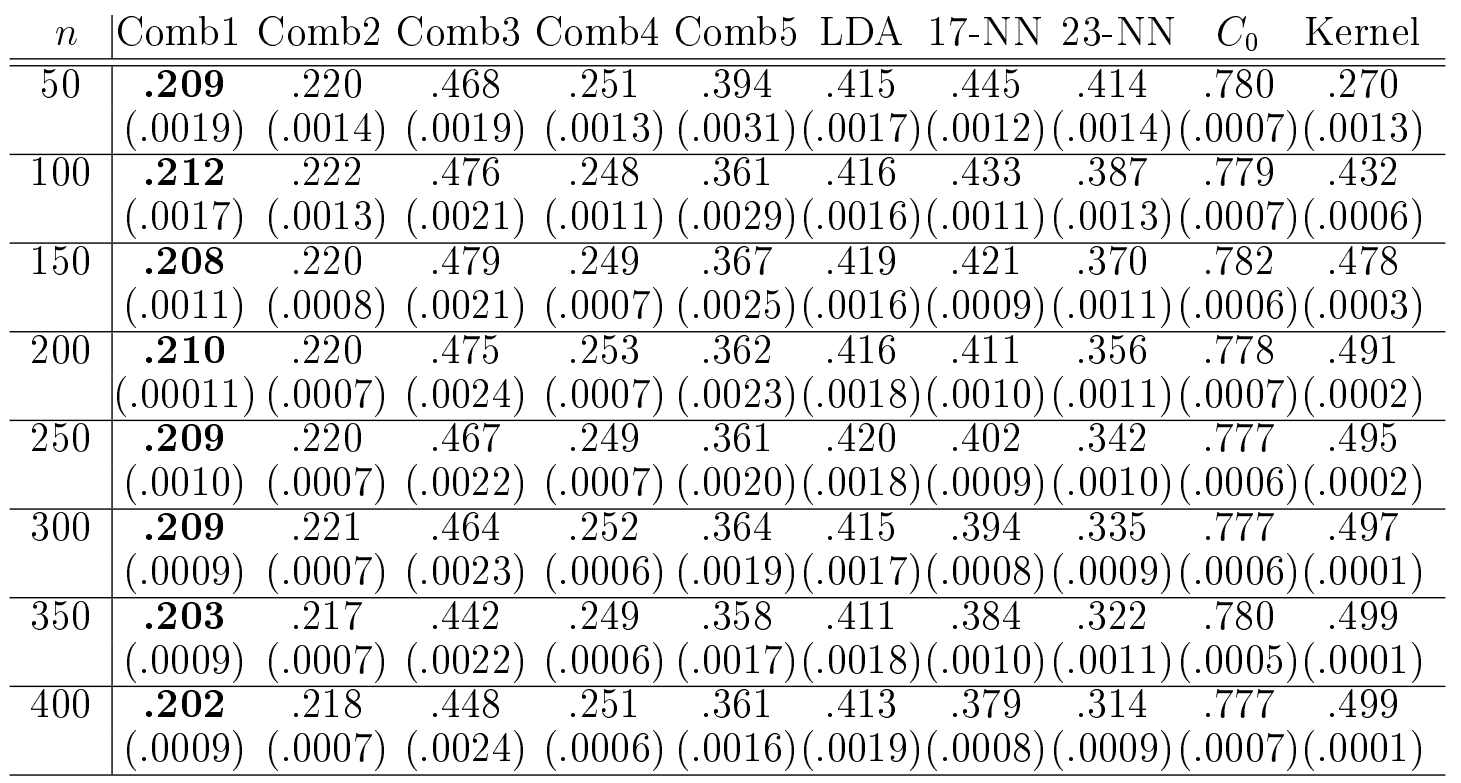

and this is particularly true for $C_{0}$ which confuses the two classes. Table 3 also shows that the benefits gained from combining these classifiers, based on Comb1 (or Comb2), are quite significant without requiring $n$ to be very large.

\section{Remarks.}

Our numerical studies indicate that: (i) combining several weak/poor classifiers can be more effective in reducing the error rate than the case where one or more of the preliminary classifiers are good performers. (ii) The more individual classifiers are included in the ensemble, the more data (i.e., larger $n$ ) will be needed for the combined classifier to work effectively. This is particularly evident from a comparison of examples A nd B, where the number of individual classifiers $M$ is changed from 6 to 3 . Although it is virtually impossible to give a precise general guideline as to when (i.e., for what values of $n$ ) the proposed combined classifier is more interesting than the initial classifiers, it is still possible to give a conservative lower bound on $n$, in terms of $M$, that would yield a more effective combined classifier. In 
fact, the proof of Theorem 2 can be used to show that if $M=o(\log n)$ or, from a practical point of view, if $n$ is of order $e^{M}$ (or larger), then one should expect the combined classifier to become more interesting than the individual ones. We believe that in most situations, this conservative lower bound on $n$ is much larger than what is needed in practice. For example, with 6 individual classifiers in Example A, the combined classifier is already performing quite well with just $n=150$ (instead of $n=e^{6}$ which is about 400). To appreciate the above bound, observe that in the notation of Theorem 2 and its proof, $\mathcal{R}\left(\widehat{\phi}_{n}\right)-\mathcal{R}\left(\phi_{n}^{*}\right):=\Delta_{n, 1}+\Delta_{n, 2}$, where $\Delta_{n, 1}$ and $\Delta_{n, 2}$ are as in (9). But the exponential bound in (13) can be used to establish the loose upper bound $\Delta_{n, 1} \leq \sqrt{\log \left(2^{M+2}\right) 2^{2 M+6} /(2 \ell)}+\sqrt{2^{2 M+6} /\left(8 \ell \log \left(2^{M+2}\right)\right)}$. Now, since $\ell=\alpha n$ for some $\alpha \in(0,1)$, it is not hard to see that, approximately, $M=o(\log n)$, which yields $n \geq e^{M}$.

\section{Appendix}

\section{PROOF OF THEOREM 1}

To prove the theorem we show that for any other classifier $\phi:\{0,1\}^{M} \rightarrow\{0,1\}$, which may also depend on the data, $P\left\{\phi_{n}^{*}\left(\widehat{\boldsymbol{\psi}}_{n}(\mathbf{X})\right) \neq Y\right\} \leq P\left\{\phi\left(\widehat{\boldsymbol{\psi}}_{n}(\mathbf{X})\right) \neq Y\right\}$. Now observe that

$$
\begin{aligned}
P & \left\{\phi_{n}^{*}\left(\widehat{\boldsymbol{\psi}}_{n}(\mathbf{X})\right) \neq Y\right\}-P\left\{\phi\left(\widehat{\boldsymbol{\psi}}_{n}(\mathbf{X})\right) \neq Y\right\} \\
& =P\left\{\phi\left(\widehat{\boldsymbol{\psi}}_{n}(\mathbf{X})\right)=Y\right\}-P\left\{\phi_{n}^{*}\left(\widehat{\boldsymbol{\psi}}_{n}(\mathbf{X})\right)=Y\right\} \\
& =\sum_{k=0,1} E\left[I\left\{\phi\left(\widehat{\boldsymbol{\psi}}_{n}(\mathbf{X})\right)=k, Y=k\right\}-I\left\{\phi_{n}^{*}\left(\widehat{\boldsymbol{\psi}}_{n}(\mathbf{X})\right)=k, Y=k\right\}\right] \\
& =\sum_{k=0,1} E\left\{E\left[\left(I\left\{\phi\left(\widehat{\boldsymbol{\psi}}_{n}(\mathbf{X})\right)=k\right\}-I\left\{\phi_{n}^{*}\left(\widehat{\boldsymbol{\psi}}_{n}(\mathbf{X})\right)=k\right\}\right) I\{Y=k\} \mid \widehat{\boldsymbol{\psi}}_{n}(\mathbf{X})\right]\right\} \\
= & E\left\{\left[I\left\{\phi\left(\widehat{\boldsymbol{\psi}}_{n}(\mathbf{X})\right)=1\right\}-I\left\{\phi_{n}^{*}\left(\widehat{\boldsymbol{\psi}}_{n}(\mathbf{X})\right)=1\right\}\right] P\left\{Y=1 \mid \widehat{\boldsymbol{\psi}}_{n}(\mathbf{X})\right\}\right\} \\
& -E\left\{\left[I\left\{\phi\left(\widehat{\boldsymbol{\psi}}_{n}(\mathbf{X})\right)=1\right\}-I\left\{\phi_{n}^{*}\left(\widehat{\boldsymbol{\psi}}_{n}(\mathbf{X})\right)=1\right\}\right]\left(1-P\left\{Y=1 \mid \widehat{\boldsymbol{\psi}}_{n}(\mathbf{X})\right\}\right)\right\} \\
= & E\left\{\left[I\left\{\phi\left(\widehat{\boldsymbol{\psi}}_{n}(\mathbf{X})\right)=1\right\}-I\left\{\phi_{n}^{*}\left(\widehat{\boldsymbol{\psi}}_{n}(\mathbf{X})\right)=1\right\}\right]\left(2 P\left\{Y=1 \mid \widehat{\boldsymbol{\psi}}_{n}(\mathbf{X})\right\}-1\right)\right\} \\
= & -2 E\left\{I\left\{\phi\left(\widehat{\boldsymbol{\psi}}_{n}(\mathbf{X})\right) \neq \phi_{n}^{*}\left(\widehat{\boldsymbol{\psi}}_{n}(\mathbf{X})\right)\right\} \times\left|P\left\{Y=1 \mid \widehat{\boldsymbol{\psi}}_{n}(\mathbf{X})\right\}-\frac{1}{2}\right|\right\} \leq 0 .
\end{aligned}
$$




\section{PROOF OF THEOREM 2}

Part (i). Let

$$
\begin{aligned}
\mathcal{R}\left(\widehat{\phi}_{n} \mid \mathbb{D}_{n}\right) & =P\left\{\widehat{\phi}_{n}\left(\widehat{\boldsymbol{\psi}}_{m}(\mathbf{X})\right) \neq Y \mid \mathbb{D}_{n}\right\} \\
\mathcal{R}\left(\phi_{n, h} \mid \mathbb{D}_{n}\right) & =P\left\{\phi_{n, h}\left(\widehat{\boldsymbol{\psi}}_{m}(\mathbf{X})\right) \neq Y \mid \mathbb{D}_{n}\right\}, \quad \forall \phi_{n, h} \in \Phi_{n},
\end{aligned}
$$

and write

$$
\begin{aligned}
\mathcal{R}\left(\widehat{\phi}_{n}\right)-\mathcal{R}\left(\phi_{n}^{*}\right)= & \left\{\mathcal{R}\left(\widehat{\phi}_{n}\right)-E\left[\inf _{\phi_{n, h} \in \Phi_{n}} \mathcal{R}\left(\phi_{n, h} \mid \mathbb{D}_{n}\right)\right]\right\} \\
& \quad+\left\{E\left[\inf _{\phi_{n, h} \in \Phi_{n}} \mathcal{R}\left(\phi_{n, h} \mid \mathbb{D}_{n}\right)\right]-\mathcal{R}\left(\phi_{n}^{*}\right)\right\} \\
:= & \Delta_{n, 1}+\Delta_{n, 2} .
\end{aligned}
$$

We will show that each term converges to zero. Let $\widehat{\mathcal{R}}_{\ell}\left(\phi_{n, h}\right)$ be as in (6) and note that

$$
\begin{aligned}
\mathcal{R}\left(\widehat{\phi}_{n} \mid \mathbb{D}_{n}\right)-\inf _{\phi_{n, h} \in \Phi_{n}} \mathcal{R}\left(\phi_{n, h} \mid \mathbb{D}_{n}\right) & =\mathcal{R}\left(\widehat{\phi}_{n} \mid \mathbb{D}_{n}\right)-\widehat{\mathcal{R}}_{\ell}\left(\widehat{\phi}_{n}\right)+\widehat{\mathcal{R}}_{\ell}\left(\widehat{\phi}_{n}\right)-\inf _{\phi_{n, h} \in \Phi_{n}} \mathcal{R}\left(\phi_{n, h} \mid \mathbb{D}_{n}\right) \\
& \leq 2 \sup _{\phi_{n, h} \in \Phi_{n}}\left|\widehat{\mathcal{R}}_{\ell}\left(\phi_{n, h}\right)-\mathcal{R}\left(\phi_{n, h} \mid \mathbb{D}_{n}\right)\right|
\end{aligned}
$$

Next, fix $\mathbb{D}_{n}$ and let $\mathcal{C}_{n, 1}$ be the class of sets of the form

$$
\left\{\widehat{\boldsymbol{\psi}}_{m}(\mathbf{x}), \text { as } \mathbf{x} \text { varies over } \mathbb{R}^{d} \mid \phi_{n, h}\left(\widehat{\boldsymbol{\psi}}_{m}(\mathbf{x})\right)=1\right\} \times\{0\}, \phi_{n, h} \in \Phi_{n} .
$$

Also, let $\mathcal{C}_{n, 0}$ be the class of sets of the form

$$
\left\{\widehat{\boldsymbol{\psi}}_{m}(\mathbf{x}), \text { as } \mathbf{x} \text { varies over } \mathbb{R}^{d} \mid \phi_{n, h}\left(\widehat{\boldsymbol{\psi}}_{m}(\mathbf{x})\right)=0\right\} \times\{1\}, \phi_{n, h} \in \Phi_{n},
$$

and note that

$$
\begin{aligned}
& \sup _{\phi_{n, h} \in \Phi_{n}}\left|\widehat{\mathcal{R}}_{\ell}\left(\phi_{n, h}\right)-\mathcal{R}\left(\phi_{n, h} \mid \mathbb{D}_{n}\right)\right| \\
& \leq \sup _{c_{n, 1} \in \mathcal{C}_{n, 1}}\left|\frac{1}{\ell} \sum_{i:\left(\mathbf{x}_{i}, Y_{i}\right) \in \mathbb{D}_{\ell}} I\left\{\left(\widehat{\boldsymbol{\psi}}_{m}\left(\mathbf{X}_{i}\right), Y_{i}\right) \in c_{n, 1}\right\}-P\left\{\left(\widehat{\boldsymbol{\psi}}_{m}(\mathbf{X}), Y\right) \in c_{n, 1} \mid \mathbb{D}_{n}\right\}\right| \\
& +\sup _{c_{n, 0} \in \mathcal{C}_{n, 0}}\left|\frac{1}{\ell} \sum_{i:\left(\mathbf{X}_{i}, Y_{i}\right) \in \mathbb{D}_{\ell}} I\left\{\left(\widehat{\boldsymbol{\psi}}_{m}\left(\mathbf{X}_{i}\right), Y_{i}\right) \in c_{n, 0}\right\}-P\left\{\left(\widehat{\boldsymbol{\psi}}_{m}(\mathbf{X}), Y\right) \in c_{n, 0} \mid \mathbb{D}_{n}\right\}\right| .
\end{aligned}
$$

Therefore,

$$
\text { r.h.s. of }(10) \leq 2 \sup _{c_{n, 1} \in \mathcal{C}_{n, 1}}\left|\mu_{\ell}\left(c_{n, 1}\right)-\mu\left(c_{n, 1} \mid \mathbb{D}_{n}\right)\right|+2 \sup _{c_{n, 0} \in \mathcal{C}_{n, 0}}\left|\mu_{\ell}\left(c_{n, 0}\right)-\mu\left(c_{n, 0} \mid \mathbb{D}_{n}\right)\right|,
$$


where, for $j=0,1$,

$$
\begin{aligned}
\mu_{\ell}\left(c_{n, j}\right) & =\frac{1}{\ell} \sum_{i:\left(\mathbf{X}_{i}, Y_{i}\right) \in \mathbb{D}_{\ell}} I\left\{\left(\widehat{\boldsymbol{\psi}}_{m}\left(\mathbf{X}_{i}\right), Y_{i}\right) \in c_{n, j}\right\} \\
\mu\left(c_{n, j} \mid \mathbb{D}_{n}\right) & =P\left\{\left(\widehat{\boldsymbol{\psi}}_{m}(\mathbf{X}), Y\right) \in c_{n, j} \mid \mathbb{D}_{n}\right\} .
\end{aligned}
$$

Now let $\mathcal{B}$ be the collection of the Borel sets of $\mathbb{R}^{M+1}$ and observe that

$$
\text { r.h.s. of }(11) \leq 4 \sup _{B \in \mathcal{B}}\left|\mu_{\ell}(B)-\mu\left(B \mid \mathbb{D}_{m}\right)\right| \leq 4 \sum_{\mathbf{u} \in\{0,1\}^{M+1}}\left|\mu_{\ell}(\{\mathbf{u}\})-\mu\left(\{\mathbf{u}\} \mid \mathbb{D}_{m}\right)\right|,
$$

where, for each $\mathbf{u} \in\{0,1\}^{M+1}, \mu_{\ell}(\{\mathbf{u}\})=\ell^{-1} \sum_{i:\left(\mathbf{X}_{i}, Y_{i}\right) \in \mathbb{D}_{\ell}} I\left\{\left(\widehat{\boldsymbol{\psi}}_{m}\left(\mathbf{X}_{i}\right), Y_{i}\right)=\mathbf{u}\right\}$ and $\mu\left(\{\mathbf{u}\} \mid \mathbb{D}_{m}\right)=$ $P\left\{\left(\widehat{\boldsymbol{\psi}}_{m}(\mathbf{X}), Y\right)=\mathbf{u} \mid \mathbb{D}_{m}\right\}$. Since, conditional on $\mathbb{D}_{m}$, the $\ell$ terms $\left(\widehat{\boldsymbol{\psi}}_{m}\left(\mathbf{X}_{i}\right), Y_{i}\right)$ corresponding to the $\ell$ pairs $\left(\mathbf{X}_{i}, Y_{i}\right) \in \mathbb{D}_{\ell}$ are independent $\{0,1\}^{M+1}$-valued random vectors, one finds (Hoeffding's (1963) inequality) that for every $\mathbf{u} \in\{0,1\}^{M+1}$ and each $\epsilon>0$,

$$
P\left\{\left|\mu_{\ell}(\{\mathbf{u}\})-\mu\left(\{\mathbf{u}\} \mid \mathbb{D}_{m}\right)\right|>\epsilon\right\}=E\left[P\left\{\left|\mu_{\ell}(\{\mathbf{u}\})-\mu\left(\{\mathbf{u}\} \mid \mathbb{D}_{m}\right)\right|>\epsilon \mid \mathbb{D}_{m}\right\}\right] \leq 2 e^{-2 \ell \epsilon^{2}} .
$$

Therefore, in view of (10), (11), and (12), for every $\epsilon>0$

$$
\begin{aligned}
& P\left\{\mathcal{R}\left(\widehat{\phi}_{n} \mid \mathbb{D}_{n}\right)-\inf _{\phi_{n, h} \in \Phi_{n}} \mathcal{R}\left(\phi_{n, h} \mid \mathbb{D}_{n}\right)>\epsilon\right\} \\
& \leq P\left\{\sup _{\phi_{n, h} \in \Phi_{n}}\left|\widehat{\mathcal{R}}_{\ell}\left(\phi_{n, h}\right)-\mathcal{R}\left(\phi_{n, h} \mid \mathbb{D}_{n}\right)\right|>\frac{\epsilon}{2}\right\} \\
& \leq P\left\{\sum_{\mathbf{u} \in\{0,1\}^{M+1}}\left|\mu_{\ell}(\{\mathbf{u}\})-\mu\left(\{\mathbf{u}\} \mid \mathbb{D}_{m}\right)\right|>\frac{\epsilon}{4}\right\} \\
& \leq \sum_{\mathbf{u} \in\{0,1\}^{M+1}} P\left\{\left|\mu_{\ell}(\{\mathbf{u}\})-\mu\left(\{\mathbf{u}\} \mid \mathbb{D}_{m}\right)\right|>\frac{\epsilon}{2^{M+3}}\right\} \leq 2^{M+2} \exp \left\{-\frac{2 \ell \epsilon^{2}}{2^{2(M+3)}}\right\} .
\end{aligned}
$$

Thus, by the Borel-Cantelli lemma, $\mathcal{R}\left(\widehat{\phi}_{n} \mid \mathbb{D}_{n}\right)-\inf _{\phi_{n, h} \in \Phi_{n}} \mathcal{R}\left(\phi_{n, h} \mid \mathbb{D}_{n}\right) \longrightarrow^{\text {a.s. }} 0$, as $n$ (and thus $\ell) \rightarrow \infty$. Therefore, by the dominated convergence theorem (DCT), as $n \rightarrow \infty$,

$$
\Delta_{n, 1} \stackrel{(9)}{=} \mathcal{R}\left(\widehat{\phi}_{n}\right)-E\left[\inf _{\phi_{n, h} \in \Phi_{n}} \mathcal{R}\left(\phi_{n, h} \mid \mathbb{D}_{n}\right)\right]=E\left[\mathcal{R}\left(\widehat{\phi}_{n} \mid \mathbb{D}_{n}\right)-\inf _{\phi_{n, h} \in \Phi_{n}} \mathcal{R}\left(\phi_{n, h} \mid \mathbb{D}_{n}\right)\right] \stackrel{\mathrm{DCT}}{\longrightarrow} 0 .
$$

Next, we show that $\Delta_{n, 2} \rightarrow 0$. Define the combined classifier $\phi_{m}^{*}$ by

$$
\phi_{m}^{*}\left(\widehat{\boldsymbol{\psi}}_{m}(\mathbf{X})\right)= \begin{cases}1 & \text { if } P\left\{Y=1 \mid \widehat{\boldsymbol{\psi}}_{m}(\mathbf{X})\right\}>\frac{1}{2} \\ 0 & \text { otherwise, }\end{cases}
$$


and let $\mathcal{R}\left(\phi_{m}^{*}\right)=P\left\{\phi_{m}^{*}\left(\widehat{\boldsymbol{\psi}}_{m}(\mathbf{X})\right) \neq Y\right\}$ be its error probability. Now write (in view of $(9)$ )

$$
\Delta_{n, 2}=\left\{E\left[\inf _{\phi_{n, h} \in \Phi_{n}} \mathcal{R}\left(\phi_{n, h} \mid \mathbb{D}_{n}\right)\right]-\mathcal{R}\left(\phi_{m}^{*}\right)\right\}+\left\{\mathcal{R}\left(\phi_{m}^{*}\right)-\mathcal{R}\left(\phi_{n}^{*}\right)\right\}:=\Delta_{n, 2, A}+\Delta_{n, 2, B},
$$

and observe that since $E\left[\inf _{\phi_{n, h} \in \Phi_{n}} \mathcal{R}\left(\phi_{n, h} \mid \mathbb{D}_{n}\right)\right] \leq E\left[\mathcal{R}\left(\phi_{n, 0} \mid \mathbb{D}_{n}\right)\right]=\mathcal{R}\left(\phi_{n, 0}\right)$, where $\mathcal{R}\left(\phi_{n, 0}\right)=$ $P\left\{\phi_{n, 0}\left(\widehat{\boldsymbol{\psi}}_{m}(\mathbf{X})\right) \neq Y\right\}$, one finds $\Delta_{n, 2, A} \leq \mathcal{R}\left(\phi_{n, 0}\right)-\mathcal{R}\left(\phi_{m}^{*}\right)$. Furthermore, since

$$
\begin{aligned}
E\left[\inf _{\phi_{n, h} \in \Phi_{n}} \mathcal{R}\left(\phi_{n, h} \mid \mathbb{D}_{n}\right)\right] & =E\left[\mathcal{R}\left(\underset{\phi_{n, h} \in \Phi_{n}}{\operatorname{argmin}} \mathcal{R}\left(\phi_{n, h} \mid \mathbb{D}_{n}\right) \mid \mathbb{D}_{n}\right)\right] \\
& =\mathcal{R}\left(\underset{\phi_{n, h} \in \Phi_{n}}{\operatorname{argmin}} \mathcal{R}\left(\phi_{n, h} \mid \mathbb{D}_{n}\right)\right) \\
& \geq \mathcal{R}\left(\phi_{m}^{*}\right),(\text { by Theorem } 1 \text { with } n \text { replaced by } m),
\end{aligned}
$$

where $\mathcal{R}\left(\phi_{m}^{*}\right)=P\left\{\phi_{m}^{*}\left(\widehat{\boldsymbol{\psi}}_{m}(\mathbf{X})\right) \neq Y\right\}$, one finds $0 \leq \Delta_{n, 2, A}$. Therefore, one has

$$
0 \leq \Delta_{n, 2, A} \leq \mathcal{R}\left(\phi_{n, 0}\right)-\mathcal{R}\left(\phi_{m}^{*}\right)
$$

Now put

$$
\begin{aligned}
Q\left(\widehat{\boldsymbol{\psi}}_{m}(\mathbf{X})\right) & =P\left\{Y=1 \mid \widehat{\boldsymbol{\psi}}_{m}(\mathbf{X})\right\} \\
\widehat{Q}_{\ell}\left(\widehat{\boldsymbol{\psi}}_{m}(\mathbf{X})\right) & =\frac{\sum_{i:\left(\mathbf{X}_{i}, Y_{i}\right) \in \mathbb{D}_{\ell}} I\left\{Y_{i}=1, \widehat{\boldsymbol{\psi}}_{m}\left(\mathbf{X}_{i}\right)=\widehat{\boldsymbol{\psi}}_{m}(\mathbf{X})\right\}}{N_{m, \ell}(\mathbf{X})}
\end{aligned}
$$

where $N_{m, \ell}(\mathbf{X})$ is as in (5). Then it can be shown (see the Appendix) that

$$
\mathcal{R}\left(\phi_{n, 0}\right)-\mathcal{R}\left(\phi_{m}^{*}\right) \leq 2 E\left|\widehat{Q}_{\ell}\left(\widehat{\boldsymbol{\psi}}_{m}(\mathbf{X})\right)-Q\left(\widehat{\boldsymbol{\psi}}_{m}(\mathbf{X})\right)\right|
$$

Therefore,

$$
\Delta_{n, 2} \leq 2 E\left|\widehat{Q}_{\ell}\left(\widehat{\boldsymbol{\psi}}_{m}(\mathbf{X})\right)-Q\left(\widehat{\boldsymbol{\psi}}_{m}(\mathbf{X})\right)\right|+\left(\mathcal{R}\left(\phi_{m}^{*}\right)-\mathcal{R}\left(\phi_{n}^{*}\right)\right)
$$

To show that the above expectation converges to zero, as $n \rightarrow \infty$, we employ some of the arguments used by Devroye et al. (1996, Sec. 6.3). Let $\mathcal{I}_{\ell}$ be the index set of the observations in $\mathbb{D}_{\ell}$, i.e., the set $\mathcal{I}_{\ell}=\left\{i \in\{1, \ldots, n\} \mid\left(\mathbf{X}_{i}, Y_{i}\right) \in \mathbb{D}_{\ell}\right\}$ and note that, conditional on $\mathbb{D}_{m}, \mathbf{X}$, and the random variables $I\left\{\widehat{\boldsymbol{\psi}}_{m}\left(\mathbf{X}_{i}\right)=\widehat{\boldsymbol{\psi}}_{m}(\mathbf{X})\right\}, i \in \mathcal{I}_{\ell}$, the random variable $N_{m, \ell}(\mathbf{X}) \times \widehat{Q}_{\ell}\left(\widehat{\boldsymbol{\psi}}_{m}(\mathbf{X})\right)$ has a Binomial $\left(N_{m, \ell}(\mathbf{X}), Q\left(\widehat{\boldsymbol{\psi}}_{m}(\mathbf{X})\right)\right)$ distribution, where the probability of success $Q\left(\widehat{\boldsymbol{\psi}}_{m}(\mathbf{X})\right)$ is given by (16). Now observe that

$$
E\left|\widehat{Q}_{\ell}\left(\widehat{\boldsymbol{\psi}}_{m}(\mathbf{X})\right)-Q\left(\widehat{\boldsymbol{\psi}}_{m}(\mathbf{X})\right)\right|
$$




$$
\leq\left(E\left[E\left(\left|\widehat{Q}_{\ell}\left(\widehat{\boldsymbol{\psi}}_{m}(\mathbf{X})\right)-Q\left(\widehat{\boldsymbol{\psi}}_{m}(\mathbf{x})\right)\right|^{2} \mid \mathbf{X}, \mathbb{D}_{m}, I\left\{\widehat{\boldsymbol{\psi}}_{m}\left(\mathbf{X}_{i}\right)=\widehat{\boldsymbol{\psi}}_{m}(\mathbf{X})\right\}, i \in \mathcal{I}_{\ell}\right)\right]\right)^{\frac{1}{2}}
$$

(via conditioning and the Cauchy-Schwartz inequality)

$$
\begin{aligned}
\leq & \left(E \left[E \left(\left|\frac{N_{m, \ell}(\mathbf{X}) \cdot \widehat{Q}_{\ell}\left(\widehat{\boldsymbol{\psi}}_{m}(\mathbf{X})\right)}{N_{m, \ell}(\mathbf{X})}-Q\left(\widehat{\boldsymbol{\psi}}_{m}(\mathbf{X})\right)\right|^{2} \times\right.\right.\right. \\
& \left.\left.\left.I\left\{N_{m, \ell}(\mathbf{X}) \neq 0\right\} \mid \mathbf{X}, \mathbb{D}_{m}, I\left\{\widehat{\boldsymbol{\psi}}_{m}\left(\mathbf{X}_{i}\right)=\widehat{\boldsymbol{\psi}}_{m}(\mathbf{X})\right\}, i \in \mathcal{I}_{\ell}\right)+I\left\{N_{m, \ell}(\mathbf{X})=0\right\}\right]\right)^{\frac{1}{2}} \\
= & \left(E \left[E \left(\left|\frac{\text { Binomial }\left(N_{m, \ell}(\mathbf{X}), Q\left(\widehat{\boldsymbol{\psi}}_{m}(\mathbf{X})\right)\right)}{N_{m, \ell}(\mathbf{X})}-Q\left(\widehat{\boldsymbol{\psi}}_{m}(\mathbf{X})\right)\right|^{2} \times\right.\right.\right. \\
& \left.\left.\left.I\left\{N_{m, \ell}(\mathbf{X}) \neq 0\right\} \mid \mathbf{X}, \mathbb{D}_{m}, I\left\{\widehat{\boldsymbol{\psi}}_{m}\left(\mathbf{X}_{i}\right)=\widehat{\boldsymbol{\psi}}_{m}(\mathbf{X})\right\}, i \in \mathcal{I}_{\ell}\right)\right]+P\left\{N_{m, \ell}(\mathbf{X})=0\right\}\right)^{\frac{1}{2}} \\
\leq & \left(E\left[\frac{\left[\left(\widehat{\boldsymbol{\psi}}_{m}(\mathbf{X})\right)\left[1-Q\left(\widehat{\boldsymbol{\psi}}_{m}(\mathbf{X})\right)\right]\right.}{N_{m, \ell}(\mathbf{X})} \times I\left\{N_{m, \ell}(\mathbf{X}) \neq 0\right\}\right]+P\left\{N_{m, \ell}(\mathbf{X})=0\right\}\right)^{\frac{1}{2}}
\end{aligned}
$$

(in view of the binomial variance formula)

$$
\leq\left(E\left[\frac{I\left\{N_{m, \ell}(\mathbf{X}) \neq 0\right\}}{4 N_{m, \ell}(\mathbf{X})}\right]+P\left\{N_{m, \ell}(\mathbf{X})=0\right\}\right)^{\frac{1}{2}}
$$

Let $L>1$ be arbitrary and note that the expectation in (20) can be rewritten as

$$
\begin{aligned}
E\left[\frac{I\left\{N_{m, \ell}(\mathbf{X}) \neq 0\right\}}{4 N_{m, \ell}(\mathbf{X})}\right] & =E\left[\frac{I\left\{1 \leq N_{m, \ell}(\mathbf{X}) \leq L\right\}+I\left\{N_{m, \ell}(\mathbf{X})>L\right\}}{4 N_{m, \ell}(\mathbf{X})}\right] \\
& \leq P\left\{N_{m, \ell}(\mathbf{X}) \leq L\right\}+L^{-1} .
\end{aligned}
$$

Choosing $L$ large enough makes the term $L^{-1}$ as small as desired in the last expression above. Furthermore, since $N_{m, \ell}(\mathbf{X}) \rightarrow \infty$, in probability, as $\ell \rightarrow \infty$ (by assumption (A2)), the term $P\left\{N_{m, \ell}(\mathbf{X}) \leq L\right\}$ in the above expression and the term $P\left\{N_{m, \ell}(\mathbf{X})=0\right\}$ in $(20)$ can be made small by choosing $n$ (and thus $\ell$ ) large enough. Finally, since $\mathcal{R}\left(\phi_{m}^{*}\right)-\mathcal{R}\left(\phi_{n}^{*}\right) \rightarrow 0$, as $n \rightarrow \infty$ (by assumption (A3)), the bound in (19) yields $\Delta_{n, 2} \rightarrow 0$, as $n \rightarrow \infty$, and this completes the proof of part (i) of the theorem.

Part (ii). Let $\widehat{\psi}_{n, j}$ be the $j$-th individual classifier, $j=1, \ldots, M$. Also, let $\phi_{n}^{*}$ be the classifier in (2) and note that $\mathcal{R}\left(\widehat{\phi}_{n}\right)-\mathcal{R}\left(\widehat{\psi}_{n, j}\right)=\left(\mathcal{R}\left(\widehat{\phi}_{n}\right)-\mathcal{R}\left(\phi_{n}^{*}\right)\right)+\left(\mathcal{R}\left(\phi_{n}^{*}\right)-\mathcal{R}\left(\widehat{\psi}_{n, j}\right)\right)$. Taking the limsup, the first term above converges to zero by part (i). The result now follows by Theorem 1 and the fact that $j \in\{1, \ldots, M\}$ was arbitrary. 
PROOF OF (18).

As in the proof of Theorem 1, one can show that

$$
\begin{aligned}
\mathcal{R}\left(\phi_{n, 0}\right)-\mathcal{R}\left(\phi_{m}^{*}\right) & =2 E\left[\left|Q\left(\widehat{\boldsymbol{\psi}}_{m}(\mathbf{X})\right)-\frac{1}{2}\right| \cdot I\left\{\phi_{m}^{*}\left(\widehat{\boldsymbol{\psi}}_{m}(\mathbf{X})\right) \neq \phi_{n, 0}\left(\widehat{\boldsymbol{\psi}}_{m}(\mathbf{X})\right)\right\}\right] \\
& \leq 2 E\left|\widehat{Q}_{\ell}\left(\widehat{\boldsymbol{\psi}}_{m}(\mathbf{X})\right)-Q\left(\widehat{\boldsymbol{\psi}}_{m}(\mathbf{X})\right)\right|
\end{aligned}
$$

where the last line follows from the fact that, in view of the definitions of $\phi_{n, 0}$ and $\phi_{n}^{*}$ in (4) and (14), the inequality $\left|Q\left(\widehat{\boldsymbol{\psi}}_{m}(\mathbf{X})\right)-1 / 2\right| \leq\left|\widehat{Q}_{\ell}\left(\widehat{\boldsymbol{\psi}}_{m}(\mathbf{X})\right)-Q\left(\widehat{\boldsymbol{\psi}}_{m}(\mathbf{X})\right)\right|$ holds on the set $\left\{\phi_{m}^{*}\left(\widehat{\boldsymbol{\psi}}_{m}(\mathbf{X})\right) \neq \phi_{n, 0}\left(\widehat{\boldsymbol{\psi}}_{m}(\mathbf{X})\right)\right\}$.

\section{Acknowledgements:}

This work is supported by the NSF Grant DMS-1407400 of Majid Mojirsheibani.

\section{References}

Adler, W., Brenning, A., Potapov, S., and Schmid, M., and Lausen, B. (2011) Ensemble classification of paired data. Computational Statistics \& Data Analysis, 55, 1933-1941.

Balakrishnan, N. and Mojirsheibani, M. (2015) A simple method for combining estimates to improve the overall error rates in classification. Computational Statistics, 30, 1033-1049.

Biau, G., Devroye, L. and Lugosi, G. (2008) Consistency of random forests and other averaging classifiers. Journal of Machine Learning Research, 9, 2015-2033.

Biau, G., Fischer, A., Guedj, B. and Malley, J. D. (2016) COBRA: A combined regression strategy. Journal of Multivariate Analysis, 146, 18-28 (with supplementary material).

Boser, B., Guyon, I., and Vapnik, V . (1992). A training algorithm for optimal margin classifiers. In Proceedings of the Fifth Annual Workshop on Computational Learning Theory, Vol. 5, pp. 144 - 152, 1992.

Breiman, L. (1995) Stacked regression. Machine Learning, 24, 49-64.

Breiman, L. (1996) Bagging predictors. Machine Learning, 24, 123-140.

Breiman, L. (2001) Random Forests. Machine Learning, 45, 5-32.

De Bock, K.W., Coussement, K., and Van den Poel, D. (2010) Ensemble classification based on generalized additive models. Computational Statistics $\&$ Data Analysis, 54, 15351546. 
Devroye, L., Györfi, L., and Lugosi, G. (1996), A Probabilistic Theory of Pattern Recognition, Springer, New York.

Hoeffding, W. (1963), Probability Inequalities for Sums of Bounded Random Variables. Journal of the American Statistical Association, 58, 13-30.

LeBlanc, M. and Tibshirani, R. (1996) Combining estimates in regression and classification. Journal of the American Statistical Association, 91, 1641-1650.

Lin, Y. and Jeon, Y. (2006) Random forests and adaptive nearest neighbors. Journal of the American Statistical Association, 101, 578-590.

Mojirsheibani, M. (1999) Combining classifiers based on discretization. Journal of the American Statistical Association, 94, 600-609.

Mojirsheibani, M. and Montazeri, Z. (2015) Aggregating classifiers via Rademacher-Walsh polynomials. Journal of Statistical Computation and Simulation, 85, 1187-1199.

Rokach, L. (2009) Taxonomy for characterizing ensemble methods in classification tasks: a review and annotated bibliography. Computational Statistics 85 Data Analysis, 53, 4046-4072.

Rokach, L. (2010) Ensemble-based classifiers. Artificial Intelligence Review, 33, 1-39.

van der Laan, M., Polley, E., and Hubbard, A. (2007) Super learner. Statistical Applications in Genetics and Molecular Biology, 6, Art. 25, 23 pp.

Wolpert, D. (1992) Stacked Generalization. Neural Networks, 5, 241-259.

Xu, L., Kryzak, A., and Suen, C.Y. (1992) Methods of combining multiple classifiers and their applications to handwriting recognition. IEEE Transactions on Systems, Man and Cybernetics, 22, 418-435.

Yang, Y. (2004) Aggregating regression procedures to improve performance. Bernoulli, 10, 25-47.

Yang, Y. (2000) Combining different procedures for adaptive regression. Journal of Multivariate Analysis, 74, 135-161.

Zhang, C.X. and Duin, R.P.W. (2011) An experimental study of one- and two-level classifier fusion for different sample sizes. Pattern Recognition Letters, 32, 1756-1767. 